\title{
On singularities, horizons, invariants, and the results of Antoci, Liebscher and Mihich (GRG 38, 15 (2006) and earlier)
}

\author{
Malcolm A.H. MacCallum*
}

\begin{abstract}
Antoci et al. have argued that the horizons of the boost-rotation, Kerr and Schwarzschild solutions are singular, having shown that a certain invariantly-defined acceleration scalar blows up at the horizons. Their examples do not satisfy the usual definition of a singularity. It is argued that using the same term is seriously misleading and it is shown that such divergent functions are natural concomitants of regular horizons. In particular it is noted that the divergence is given by the special relativistic approximation to the overall metric. Earlier work on characterization of horizons by invariants is revisited, a new invariant criterion for them is proposed, and the relation of the acceleration invariant to the Cartan invariants, which are finite at the horizons and completely determine the spacetimes, is examined for the C-metric, Kerr and Schwarzschild cases. An appendix considers coordinate identifications at axes and horizons.
\end{abstract}

\section{Introduction}

In a recent paper Antoci et al. 4] argue that the bifurcate horizon in boostrotation metrics is singular in an invariant sense. Their argument follows very closely one which they used for the Kerr, Schwarzschild and other solutions in earlier papers [1, 2, 3], and which is also mentioned in an unpublished preprint [5] and the editorial comments by two of them on Schwarzschild's original paper 6]. In [4] they summarize it as proving that "a local, invariant, intrinsic singularity occurs when approaching the horizon" 1 .

\footnotetext{
${ }^{*}$ School of Mathematical Sciences, Queen Mary, University of London, Mile End Road, London E1 4NS, U.K. Email: m.a.h.maccallum@qmul.ac.uk

${ }^{1}$ It would seem from the cited papers that this is only one of the reasons that Antoci et al. think that the Schwarzschild solution cannot, or perhaps should not, be extended
} 
The claim is based on the fact that in each case the acceleration of a timelike unit vector field tangent to the orbits of a uniquely-determined timelike Killing vector is divergent as the horizon is approached. (Actually, the limits taken may approach only the two-sphere where past and future horizons meet, as discussed in the Appendix, but I ignore this criticism in the main text.) This divergence is well-known for the Schwarzschild case, was derived for the Kerr-Newman metric [1] and the Zipoy-Voorhees or Bach and Weyl 'gamma' metric [2], and calculated approximately for the C-metric as a representative of the boost-rotation cases [4. It was also re-derived for the Schwarzschild case by taking a two-body solution due to Bach and Weyl and considering the limit as the mass of the second body tends to zero [3].

Presence of a diverging invariant is not sufficient to prove the presence of a singularity in the sense now standard in the field. In the following section I point out that the acceleration invariant of Antoci et al. does not produce a singularity in this sense, and show, from the special relativistic approximation, that a divergent acceleration is to be expected whenever one has a regular horizon. Moreover it is noted that the approximate calculation of Antoci et al. in the C-metric is an exact calculation in the manifestly nonsingular Minkowski space.

Antoci et al. [5] argue that the special relativistic analogy is inappropriate since it is of crucial importance that the vector field considered is invariantly defined and that the acceleration is therefore referred to some standard of absolute rest. I present below a contrary view.

The final section of this paper studies the relation of the acceleration invariant to the Cartan invariants which characterize the solutions but remain finite on the horizons. It has been known for some time that in the Kruskal-Szekeres spacetime, which is the maximal analytic extension of the Schwarzschild metric, the obvious scalar polynomial formed from the first derivatives of the curvature, $R^{a b c d ; e} R_{a b c d ; e}$, vanishes exactly at the horizon [7] (which illustrates the existence of invariants whose blowup does not imply a singularity: one has only to take $1 / R^{a b c d ; e} R_{a b c d ; e}$ ). I examine the link between this observation and the divergent acceleration of Antoci et al. and show that the latter is related to division by an invariant whose vanishing characterizes the horizon.

across the horizon, and the horizon should be regarded as singular. I will not go into all these other arguments: one of them is touched on in the Appendix here. 


\section{Conceptual aspects}

To discuss singularities we need first to define them. Singularities in general relativity are defined by reference to incomplete, inextendible, causal geodesics. The reasons for using this definition were elegantly and entertainingly explained by Geroch through a Galilean dialogue [8. There has been much work on the occurrence and possible natures of singularities thus

defined. For an account of this see 9] and, for a more recent review on causal structure and boundaries, 10.

It is well-known that the horizons of the Schwarzschild, Kerr-Newman and boost-rotation solutions do not fulfil this definition. In these cases, the regions discussed by Antoci et al. are isometric to regions of a larger manifold including the horizon in which every point of the horizon is regular (i.e. has a neighbourhood which is in first approximation a neighbourhood of Minkowski space). In the Schwarzschild case this larger spacetime can be taken in the well-known Kruskal-Szekeres form. Hence geodesics can be extended through and beyond the horizon, so the horizon is not singular. (Of course, there still could be, and in the Schwarzschild and Kerr cases there are, singularities inside the horizon.)

Divergence of an invariant only implies a singularity if its blow up is sufficient to prove that geodesics cannot be continued. Antoci et al. have not shown that their invariants do this, for the cases with horizons, and the results referred to above show that it is not the case. In my view, using the word 'singularity' in this context is likely to be misleading and possibly tendentious (in that it might lead an unwary reader to believe that the presence of a singularity in the usual sense has been proved).

For completeness, one should note that the cases considered by Antoci et al. in which the Kretschmann scalar blows up do fulfil the criterion for singularities at the boundaries. For instance, the general 'gamma'-metric or Zipoy-Voorhees solutions give examples of 'directional singularities' which include singular points, rather than just regular horizons (for fuller discussion of their singularity structure, see e.g. [11, 12, 13, and literature cited therein). However, the presence of these singularities is not proved by the blow-up of the acceleration invariant: the Zipoy-Voorhees family includes the Schwarzschild metric which is not singular at the horizon. (It is therefore hardly surprising that the Kretschmann scalar's behaviour is discontinuous as a function of the parameter characterizing members of this family, as noted in [2.) I do not discuss further the cases with an divergent Kretschmann scalar.

So far I have not addressed the question of whether the behaviour found 
by Antoci et al. should be regarded as singular in some sense, regardless of its relation to the usual definition. They remark [3], apropos of singular acceleration in the Schwarzschild case, that "this singular behaviour of the gravitational pull ... has not generally aroused very much concern". Taking a Newtonian analogy, as Antoci et al. do [1, citing Abrams, divergent accelerations would suggest a point source singularity. The more appropriate analogy with special relativity I now describe (and which is discussed in [5], which its authors kindly drew to my attention on reciving an earlier draft of this paper) shows that such divergent accelerations are naturally associated with any regular horizon, despite Antoci et al.'s implicit assumption to the contrary. There is thus no cause for concern.

Consider particles in special relativity. We need only consider worldlines with constant acceleration, since Killing vector trajectories must have this property. A standard exercise in special relativity shows that, in units such that $c=1$ and in pseudo-Cartesian coordinates whose $x$-axis is aligned with the acceleration, a particle initially at rest at $x=1 / b, y=z=0$, and subject to a constant acceleration $b$ has the trajectory described by

$$
x=\left(1+b^{2} t^{2}\right)^{1 / 2} / b .
$$

This curve is asymptotic to the null hyperplane $x=t$. Its acceleration is inversely proportional to the proper time, along the geodesic with the same initial position and velocity, between the initial point and the null plane.

The curve (10) is a trajectory of the Killing vector field corresponding to boosts in the $x$ direction about the origin. If we took all the trajectories of this Killing vector field, it is easy to see their accelerations become divergent as we take a sequence of trajectories approaching $x=t$, for example by taking initial points on $t=0$ with $x \rightarrow 0$ from above.

It is not surprising that the more abruptly one wants to accelerate a particle to the speed of light, the larger the acceleration needed ${ }^{2}$. Even in Newtonian theory this would be true: however, Newtonian theory gives us no reason to consider such a set of curves. Special and general relativity were introduced precisely to explain observations which had no Newtonian explanation, so it is not surprising they lead to new considerations.

The general relativistic behaviour and the manifestly non-singular special relativistic behaviour are analogous in the neighbourhood of any regular point, as a consequence of the principle of equivalence. One can make this more explicit by expanding the metric, at a point on the regular horizon,

\footnotetext{
${ }^{2}$ Strictly, of course, a massive body in relativity can travel at the speed of light only if it loses its rest mass.
} 
in Riemannian normal coordinates: only the leading flat-space term contributes to the divergence of the acceleration, the role of the lower-order curvature terms being only to pick out the invariant vector field. Any null plane (or line) in Minkowski space has a similar set of associated Killing vector trajectories, so the analogy leads to the conclusion that any smooth regular null surface in general relativity can be associated with a set of curves of divergent acceleration. In particular, when we have an invariantlydefined set of Killing vector trajectories with a regular lightlike horizon, we would expect this horizon to be accompanied by divergent acceleration of the invariantly-defined unit vector field tangent to the Killing trajectories.

In the Schwarzschild, Kerr and boost-rotation spacetimes, the extended manifolds clearly reveal the horizons to be lightlike. Therefore any massive body which does not cross them has to be accelerated so that its worldline is asymptotic to a null curve, and the special relativistic analogy just developed applies. Indeed, it is the special relativistic contribution which Antoci et al. calculate in 4], since the approximation used in their equation (10) to derive the divergence is just the specialization of their (1) which gives flat space. Hence they are using the divergence in non-singular Minkowski space in arguing that the horizons of the $\mathrm{C}$-metric are singular!

In [5] it is argued that the analogy is not appropriate because the divergent acceleration in special relativity is not an invariant. Correspondingly, the null plane asymptotic to (10) is not an invariantly-defined horizon. The Antoci et al. method, as given in their papers, is limited to cases where there is an invariantly-defined unit timelike vector field. If there is more than a one-parameter symmetry group, the argument based on acceleration of a timelike Killing vector requires a unique choice among the possible Killing vector fields e.g. by their asymptotic properties, as exemplified in [1]. We could make the special relativistic calculation (11) invariant by cutting a wedge out of Minkowski space and considering the unique timelike Killing vector field whose trajectories do not cross the boundary of the wedge, and with respect to which the wedge is static. (This is somewhat artificial, but it does use a region corresponding to the region $r>2 m$ of the Schwarzschild solution.) There is no reason to think that the invariant character of the timelike vector fields considered by Antoci et al. affects the question of whether there is a true singularity.

However, it is true that the divergent acceleration of the uniquely-defined vector field characterizes the position of the horizon. In the next section we show how this can be understood in terms of vanishing of a curvature invariant at the horizon. So it is worth considering the question of analogies a little further. An analogy should not be rejected just because it does not 
capture all the features of a situation: if it did, it would not be an analogy but an identical model. But the special relativistic approximation is more than just an analogy: the existence of such an local approximation precisely characterizes the regularity of a point in general relativity, and in this sense the diverging acceleration coupled with finiteness of curvature invariants could be said to confirm the regularity of a horizon rather than weakening the argument for it.

Antoci et al. consider the divergence of acceleration significant because it relates to an invariantly-defined notion of staticity. They do not differentiate, in this argument, between cases where the length of the static Killing vector is bounded away from zero (for which their view seems to me more defensible) and those where the length tends to zero, for which the boostsymmetric interpretation seems more natural, modelled on the trajectories of boost Killing vectors in Minkowki space (or, more generally, locally boostsymmetric solutions [14]).

The physical significance of the curves discussed by Antoci et al. is doubtful in another way. They are test body tracks (though found as limits of other curves in [3]), in that there are no masses moving on them and perturbing the gravitational field, and the test bodies are accelerated although no non-gravitational force to supply the acceleration is modelled. The consideration of the limit of the Bach-Weyl two-body solution in 3 does not in my view outweigh this criticism (in this case the second 'body' is a singularity and the non-gravitational force is supplied by a 'strut' or 'rod' along the axis).

Thus a more physical discussion would imply perturbing the solutions, and then one would lose the invariant definition of the timelike congruence on which the argument depends. This limitation might imply that the approach has nothing to say even about perturbed Schwarzschild black holes, let alone other cases where what Antoci et al. call the 'gravitational pull' lacks invariant definition. However, one would expect to be able to generalize the argument and, from the special relativistic analogy, to find an divergent acceleration: the special relativistic argument above easily extends to sets of curves of constant acceleration which are not trajectories of the same Killing vector, and seems likely to extend also to cases where the acceleration is not constant along the curves. Alternatively, the approach described in the next section may provide a better way to generalize the idea. 


\section{Cartan invariants and the acceleration invariant}

It is known (see 14, chapter 9, for a review) that the Cartan invariants completely characterize the spacetime locally (an explicit reconstruction from these invariants was given for Schwarzschild and some other solutions in [15]). The completeness of the Cartan invariants as a specification of the local geometry gives a very precise form to the remarks of Synge on the lines of 'gravitational field = curvature of space-time', which Antoci et al. call vague and appear to reject [2]. Properties of the global structure or topology which do not have local consequences are not captured by the Cartan invariants, but the former do not include the acceleration invariants of Antoci et al.

In this section I will show how the divergent acceleration invariant relates to the Cartan invariants for the Kerr, Schwarzschild and C-metric cases, in which the Cartan invariants are all finite or zero at the horizon (as, consequently, are all scalar polynomial invariants in the curvature and its derivatives). My approach to this problem was prompted by the vanishing of $R^{a b c d ; e} R_{a b c d ; e}$ at the Schwarzschild horizon. It is not in general possible to isolate the acceleration term at a general point, but it can be done for the Kerr (and, a fortiori, Schwarzschild) cases. However, even in the C-metric the effect can be isolated on the symmetry axis.

I will consider the Kerr solution in Boyer-Lindquist coordinates, with the usual coordinates for Schwarzschild as a special case, and use the coordinates of [16] for the C-metric (the latter are more convenient in some respects than the coordinates of [14], Table 18.2, or the Weyl coordinates used in 4]). Thus the metrics are

$$
\mathrm{d} s^{2}=-\frac{S \Delta}{\Sigma^{2}} \mathrm{~d} t^{2}+\frac{\Sigma^{2}}{S}\left(\mathrm{~d} \phi-\frac{2 a m r \mathrm{~d} t}{\Sigma^{2}}\right)^{2} \sin ^{2} \theta-\frac{S \mathrm{~d} r^{2}}{\Delta}+S \mathrm{~d} \theta^{2},
$$

where $S=r^{2}+a^{2} \cos ^{2} \theta, \Delta=r^{2}-2 m r+a^{2}$ and $\Sigma=S-2 m r, m>0$ and $a$ being constants, and

$$
\mathrm{d} s^{2}=\frac{1}{A^{2}(x-y)^{2}}\left(f(y) \mathrm{d} t^{2}-\frac{\mathrm{d} y^{2}}{f(y)}+f(x) \mathrm{d} \phi^{2}+\frac{\mathrm{d} x^{2}}{f(x)}\right),
$$

where $f(\xi)=\left(1-\xi^{2}\right)(1+2 A m \xi)$ and $m$ and $A$ are positive constants satisfying $2 m A>1$.

The coordinate ranges will be restricted to a region outside the horizon. In (2) the region considered is where $r>r_{0}, r_{0}$ being the larger of the roots of $\Delta=0$. This includes the Schwarzschild case, $a=0$, where the region 
is $r>2 m$. The timelike Killing vector considered by Antoci et al. is a multiple of $\left(r_{0}^{2}+a^{2}\right) \partial_{t}+a \partial_{\phi}$. In (3), the appropriate region, where $\partial_{t}$ is a timelike Killing vector, is where $-1 \leq x \leq 1$ and $-1 / 2 m A<y<-1$. The acceleration horizon is where $y \rightarrow-1$, the black hole horizon is where $y \rightarrow-1 / 2 m A$, and $|x| \rightarrow 1$ gives portions of the symmetry axis. I will not make calculations on the horizon itself, but for brevity I use 'at the horizon' to mean 'as we approach the horizon'. As well as avoiding any need for coordinate transformations, this is done because the horizon itself is a hypersurface where invariants which are generically non-zero may vanish, leading to indeterminacy, except by analytic continuation, of some quantities which are elsewhere determinate. I also do not consider the regions inside the horizons, although an analogous analysis can be given, since the objective is to compare with the acceleration invariant of Antoci et al. and acceleration would not be an appropriate term where the relevant Killing vector is spacelike.

The calculations follow the general procedure described in [14, chapter 9, utilizing the Newman-Penrose notation extended to denote spinors totally symmetrized on all dashed and on all undashed indices by, for instance,

$$
\nabla \Psi_{20^{\prime}} \equiv \nabla_{\left(A \mid X^{\prime}\right.} \Psi_{\mid B C D E)} o^{A} o^{B} O^{C} \iota^{D} \iota \iota^{-} X^{\prime}
$$

where $\left\{o^{A}, \iota^{A}\right\}$ are the usual basis spinors. Note that the value of each invariant at a given point of the manifold is independent of the coordinates used. The Cartan invariants for the Schwarzschild solution have been given in earlier work [15, 17], and a general discussion of Petrov Type D vacua has been given in [18, 19]. All type $\mathrm{D}$ vacua were explicitly classified, using Cartan invariants, by Åman [20].

At the first step, classifying the curvature, we find we have in each case a vacuum metric of Petrov type D. Choosing the frame to bring the curvature into canonical form, the only non-zero invariant is $\Psi_{2}$. In the Kerr case, $\Psi_{2}=m /(r+\mathrm{i} a \cos \theta)^{3}$, and in the C-metric, $\Psi_{2}=-m A^{3}(x-y)^{3}$. So far the frame is fixed only up to a boost and a spatial rotation, the invariances of a Petrov type D Weyl tensor. Thus no timelike vector (within the plane of the two principal null vectors) is picked out. We may incidentally note that the Kretschmann scalar can only blow up if at least one of the Cartan curvature invariants does so.

The next step is to take the first derivative of the curvature. In both the Kerr and C-metric cases, the boost can be used to choose a frame such that $\nabla \Psi_{20^{\prime}}=3 \rho \Psi_{2}=-\nabla \Psi_{31^{\prime}}=3 \mu \Psi_{2}$. With this choice $\rho=\nabla \Psi_{20^{\prime}} / 3 \Psi_{2}$ is also an invariant and is real. The vanishing of the invariantly-chosen $\nabla \Psi_{20^{\prime}}$, 
or equivalently, in the present cases, $\rho=\nabla \Psi_{20^{\prime}} / 3 \Psi_{2}$, is the criterion for a horizon in terms of invariants (at least for Petrov type $\mathrm{D}$ spacetimes). It is natural in that on the horizon the outgoing null vector must be surfaceforming and non-expanding, i.e. have $\rho=0$, since the horizon is a marginally trapped surface.

Since $\nabla \Psi_{20^{\prime}}=-\nabla \Psi_{31^{\prime}}$ are the only non-zero first derivatives for Schwarzschild, it follows that in the Schwarzschild case the vanishing of $R^{a b c d ; e} R_{a b c d ; e}$ is an equivalent condition. However in general type $\mathrm{D}$ metrics, $\nabla \Psi_{21^{\prime}}=$ $3 \tau \Psi_{2}$ and $\nabla \Psi_{30^{\prime}}=-3 \pi \Psi_{2}$ will also be non-zero at a general point, and remain finite at the horizon (in Kerr they are zero at the poles). This explains Skea's finding that results similar to those of [7] do not obtain for scalar polynomials in other cases 21. Since it answers one of the other arguments sometimes made about the Schwarzschild and Kerr solutions, it is worth noting that the Cartan invariants' values show that the only essential constants are $m$, and $m$ and $a$ respectively (in the usual notation).

When $\tau \neq 0$, it is always possible to use the so-far undetermined 'spin' (the rotation in the plane perpendicular to the principal null directions of the Weyl tensor) to adjust the argument of $\tau$ : this can be done so that $\tau=-\bar{\tau}=-\pi$ in Kerr and $\tau=\bar{\tau}=-\pi$ in the C-metric. The resulting values are then invariants (the moduli are invariant whatever the argument, of course).

The criterion above for the horizon implies we can easily generate invariants which are divergent at the horizon, the simplest being $1 / \nabla \Psi_{20^{\prime}}$. Others are $\Psi_{2} / \nabla \Psi_{20^{\prime}}$, or, when $\nabla \Psi_{21^{\prime}} \neq 0, \nabla \Psi_{21^{\prime}} / \nabla \Psi_{20^{\prime}}$. My objective is to extract the particular invariant used by Antoci et al.

The equality $\nabla \Psi_{20^{\prime}}=-\nabla \Psi_{31^{\prime}}$ implies that the derivative of $\Psi_{2}$ is orthogonal to the timelike vector of the orthonormal frame associated with the Newman-Penrose one in the usual way (i.e. as in [14], (3.12)), so this vector is orthogonal to the surfaces on which $\Psi_{2}$ is constant. It is invariantly determined in the cases considered (and can be similarly fixed in all type $\mathrm{D}$ vacua, except those with local boost isotropy), and when there is a hypersurfaceorthogonal Killing vector (the C-metric and Schwarzschild cases) the two vectors will be parallel. In the Kerr solution, there is no hypersurfaceorthogonal Killing vector, and the correspondingly chosen timelike vector is parallel to the vector $\left(r^{2}+a^{2}\right) \partial_{t}+a \partial_{\phi}$ which is asymptotic at the horizon $r=r_{0}$ to the Killing vector field used by Antoci et al. [1].

The invariants from the curvature and its first derivative do not give the timelike vector's derivatives, such as the acceleration which is given by the combination of spin coefficients $(\epsilon+\bar{\epsilon}+\gamma+\bar{\gamma}) / \sqrt{2}$. Generic arguments have placed an upper bound of three derivatives on the classification pro- 
cess for type D vacua [18, 19, but it is known that at most two are actually needed 20]. We thus expect the acceleration's value to appear, in the Cartan scalars, in the second derivatives. Where this occurs is somewhat obscured in the GHP formalism [18, 19] since the crucial spin coefficients are hidden inside the differential operators. Using the Newman-Penrose form, the reason that the divergence of $\epsilon+\bar{\epsilon}$ and $\gamma+\bar{\gamma}$ does not show up in divergence of any Cartan invariants in the present cases is clear: they always arise in products with the terms $\nabla \Psi_{20^{\prime}}$ and $\nabla \Psi_{31^{\prime}}$ which vanish at the horizon.

We have no reason to expect that the expressions for the Cartan invariants can be algebraically solved for the spin coefficients in general. They would of course still be specified by the Cartan invariants, but indirectly by specifying the basis vectors whose commutators give the spin coefficients. However, for Petrov type D spacetimes with $\nabla \Psi_{20^{\prime}}=\nabla \Psi_{31^{\prime}}$ and $\nabla \Psi_{21^{\prime}}=-\nabla \Psi_{30^{\prime}}$, there is a combination of the second derivative invariants which involves the acceleration. Using the relations found for the first derivatives,

$$
\begin{aligned}
Q & \equiv 2 \nabla^{2} \Psi_{31^{\prime}}+3\left(\nabla^{2} \Psi_{42^{\prime}}-\nabla^{2} \Psi_{40^{\prime}}-\nabla^{2} \Psi_{22^{\prime}}+\nabla^{2} \Psi_{20^{\prime}}\right) / 4 \\
& =((\beta+\bar{\beta})-(\alpha+\bar{\alpha}+\pi+\bar{\pi})) \nabla \Psi_{21^{\prime}}-(\gamma+\bar{\gamma}+\epsilon+\bar{\epsilon}+2 \rho) \nabla \Psi_{20^{\prime}} .
\end{aligned}
$$

Note that specific values have not been substituted here: to do so would obscure the logic because multiples of the same values may arise in more than one way (e.g. both from partial derivatives and from spin coefficients multiplying the terms differentiated).

In the Kerr case, $\alpha, \beta$ and $\pi$ are imaginary. Thus the acceleration invariant is given by the combination of Cartan invariants $Q / \nabla \Psi_{20^{\prime}}+2 \nabla \Psi_{20^{\prime}} / 3 \Psi_{2}$. For the C-metric there seems to be no such neat way to remove the $\nabla \Psi_{21^{\prime}}$ terms from $Q$ or any similar expression at a generic point, without using values which cannot be directly algebraically deduced from the Cartan invariants (e.g. the value of $\beta+\bar{\beta}-\alpha-\bar{\alpha}$ ). It remains true, of course, that $Q / \nabla \Psi_{20^{\prime}}$ blows up at the horizon.

To directly obtain information about the acceleration from the Cartan invariants in the $\mathrm{C}$-metric, as distinct from inferring it from an integration giving the tetrad commutators, we thus have to be more subtle. The behaviour, if not the specific value, of the acceleration we seek must be independent of $x$. The invariant $\nabla \Psi_{21^{\prime}}$ must vanish at the axis of symmetry. So we could use the same combination of invariants as in the Kerr case but evaluate it at or as we approach ${ }^{3}$ the symmetry axis defined by $\nabla \Psi_{21^{\prime}} \rightarrow 0$.

\footnotetext{
${ }^{3}$ The wording here is chosen to cover the case where the axis itself is a conical singu-
} 
Note in particular that in both this and the Kerr case, as we approach certain points on the horizon all first derivative Cartan invariants vanish but the second derivative ones do not.

Thus we have found an invariant criterion for locating the horizon, at least in type D spacetimes, which reflects the fact that the horizon is a non-expanding null surface. We have then shown that the divergence of the acceleration at the horizon can be derived from non-singular invariants by a division by the invariant which characterizes the horizon by vanishing there. This entirely agrees with, and for these cases gives precise expression to, the arguments of the previous section for expecting a blow up in an acceleration invariant as the horizon is approached. This agreement, and the natural interpretability of the criteria, gives strong reasons for expecting the same ideas to carry over to any algebraically general or non-vacuum solution with analogous horizon structure. The C-metric case shows that these ideas are unlikely to be implementable in the simple form of algebraic expressions for the acceleration in terms of Cartan invariants at a general point.

\section{Acknowledgements}

I am grateful to Profs. W.B. Bonnor and J.M.M. Senovilla for advice and references, and to the principal authors of SHEEP/CLASSI (Inge Frick and Jan Åman) which was used to check some of the calculations.

\section{References}

[1] Antoci, S., Liebscher, D.-E., Mihich, L.: Astron. Nachr. 322, 137 (2001)

[2] Antoci, S., Liebscher, D.-E., Mihich, L.: Astron. Nachr. 324, 485 (2003)

[3] Antoci, S., Liebscher, D.-E., Mihich, L.: Class. Quant. Grav. 18, 3463 (2001)

[4] Antoci, S., Liebscher, D.-E., Mihich, L.: Gen. Rel. Grav. 38, 15 (2006)

[5] Antoci, S., Liebscher, D.-E., Mihich, L.: gr-qc/0308005 version 3 (2005).

[6] Antoci, S., Liebscher, D.-E.: Gen. Rel. Grav. 35, 945 (2003)

[7] Karlhede, A., Lindström, U., Åman, J.E.: Gen. Rel. Grav. 14, 569 (1982)

larity. The relation between the interpretations of $f(x)=0$ as an axis and $f(y)=0$ as a horizon is explained by the discussion in the Appendix. 
[8] Geroch, R.: Ann. Phys. 48, 526 (1968)

[9] Tipler, F.J., Clarke, C.J.S., Ellis, G.F.R.: In General Relativity and Gravitation: one hundred years after the birth of Albert Einstein, volume 2. Ed. A. Held. Plenum (1980)

[10] Garcia-Parrado, A., Senovilla, J.M.M.: Class. Quant. Grav. 22, R1-R84 (2005)

[11] Taylor, J.P.W.: Class. Quant. Grav. 22, 4961 (2005)

[12] Taylor, J.P.W.: Ph. D. thesis, Queen Mary, University of London (2006)

[13] Kodama, H., Hikida, W.: Class. Quant. Grav. 20, 5121 (2003)

[14] Stephani, H., Kramer, D., MacCallum, M.A.H., Hoenselaers, C.A., Herlt, E.: Exact solutions of Einstein's field equations, second edition. Cambridge University Press; Cambridge (2003)

[15] Karlhede, A., Lindström, U.: Gen. Rel. Grav. 15, 597 (1983)

[16] Hong, K., Teo, E.: Class. Quant. Grav. 20, 3269 (2003)

[17] Paiva, F.M., Rebouças, M.J., MacCallum, M.A.H.: Class. Quant. Grav. 10, 1165 (1993)

[18] Collins, J.M., d'Inverno, R A., and Vickers, J.A.: Class. Quant. Grav. 7, 2005 (1990)

[19] Collins, J.M., d'Inverno, R A., and Vickers, J.A.: Class. Quant. Grav. 8, L.215 (1991)

[20] Åman, J.E.: In Classical General Relativity (Proceedings of the 1983 London conference on Classical (non-quantum) General Relativity), ed. W.B. Bonnor, J.N. Islam, and M.A.H. MacCallum. Cambridge University Press; Cambridge (1984)

[21] Skea, J.E.F.: Ph. D. thesis, Sussex University (1986)

[22] Hawking, S.W., King, A.R., McCarthy, P.J.: J. Math. Phys. 17, 174 (1976)

[23] Fullwood, D.T.: J. Math. Phys. 33, 2232 (1992) 


\section{Appendix: coordinates, axes and horizons}

Axes of symmetry and the horizons considered in this paper have in common that they occur where, in some commonly-used coordinate chart, a metric component tends to zero ${ }^{4}$ (and another to infinity). For brevity, I call the system where this zero appears the 'original system' of coordinates. The axes and horizons in these cases can all be seen to consist of regular points of spacetime by using an appropriate coordinate system, which I will call the 'regular system'. However, if the original system is considered to include its boundary, the transformation between the systems usually becomes in some way pathological there, though smooth at other points. The pathology may be that some function in the transformation blows up, or that the map is many-one.

That these pathologies do not in themselves make the change unacceptable is easily seen from the example of the Euclidean plane, taking polar coordinates as the original chart with metric

$$
\mathrm{d} s^{2}=\mathrm{d} r^{2}+r^{2} \mathrm{~d} \theta^{2},
$$

where $0<r<\infty$ and $\theta$ and $\theta+2 \pi$ are identified (or where $0 \leq \theta \leq 2 \pi$ ). I will not bother about the inconsistency that coordinate maps are required to be $1-1$, while the standard way of using $\theta$ is not, as this is easily dealt with by using two charts, e.g. $-\pi<\theta<\pi$ and $0<\theta<2 \pi$, for the angular variable. Here $g_{\theta \theta} \rightarrow 0$ as $r \rightarrow 0$. The transformation to Cartesians (the regular chart), $x=r \cos \theta, y=r \sin \theta$ is many to one if continued to $r=0$, since all values of $\theta$ are mapped to the single point $x=y=0$. Nobody argues that this indicates anything strange about the Euclidean plane.

One way of dealing with these apparently problematic situations is to insist on the basic definition of a differential manifold, so that coordinate chart neighbourhoods have to be open and their boundary points are not included. (Recall that coordinate chart neighbourhoods in a manifold are defined to be open sets in 1-1 correspondence with open sets in $\mathbb{R}^{n}$.) Then the transformations are not many-one and may contain divergent functions without any departure from the usual manifold definitions, so nothing pathological happens. The axis, or horizon, is then only covered by the regular chart (which now has a perfectly satisfactory transformation to the original chart at all points in their overlap). In the example above, this means we stick to $r>0$ in ([6), and only treat $x=0=y$ in Cartesians or other coordinates smoothly related to them at the origin.

\footnotetext{
${ }^{4}$ The analogy is particularly strong in the C-metric, where both part of the axis and the acceleration horizon are at the zero $f(-1)=0$ of the $f$ in (3).
} 
An alternative approach is to consider the need for compatibility of the coordinate topology and the metric topology. (This compatibility is mentioned in [5] but its implications are not fully pursued.) If we consider a manifold endowed in the usual way with a smooth Riemannian metric, the two topologies agree on open sets, including chart neighbourhoods. The problems mentioned above arise when one tries to go from open chart neighbourhoods to neighbourhoods with boundaries (or even corners). For a manifold with boundary, the coordinate neighbourhoods are open sets in the halfspace, $H^{n}$ say, and the boundary has (locally) the topology of $\mathbb{R}^{n-1}$, from the coordinate point of view (call this the 'coordinate topology'). Trying to make this extension, we find that for axes or horizons it is not acceptable, because the two topologies no longer agree.

In the coordinate topology, for the Euclidean space example above, the problem is seen when we assume the coordinate range of $r$ is $0 \leq r<\infty$. What goes wrong at $r=0$ in the semi-infinite space $H^{2}$ in (6) is that the usual metric topology is no longer Hausdorff: it is impossible to take two non-overlapping open sets, one containing $\left(0, \theta_{1}\right)$ and the other containing $\left(0, \theta_{1}\right)$, for $\theta_{1} \neq \theta_{2}$. To make the topologies consistent we have to identify all the points $(0, \theta)$, and the coordinate transformation becomes many-one.

The metric topology for positive-definite Riemannian manifolds is well understood, and it resolves the questions about axes (indeed can be extended to take care of the cases where the axis is a conical singularity with an angle defect, see e.g. [16]). In particular, it deals with the C-metric at $|x| \rightarrow 1$, and the Kerr and Schwarzschild solutions at the points where $\sin \theta=0$.

The choice of an appropriate topology for indefinite (pseudo-)Riemannian manifolds is less widely discussed, and indeed more than one has been proposed [22, 23]. However, they give the same conclusions for the horizons under discussion. To be specific we could take the Alexandrov topology. To illustrate the outcome, consider the Schwarzschild solution in the form of (2) with $a=0$. If we wish to take $r \geq 2 m$ rather than $r>2 m$ we have to consider the metric topology at $r=2 m$ for all $t, \theta$, and $\phi$.

The consistency of topologies requirement is satisfied by the KruskalSzekeres picture, in which different values of $\theta$ and $\phi$ at $r=2 m$ remain distinct. This is consistent with the behaviour of the three-dimensional positive-definite metric topology of the surfaces $t=$ constant. A few authors have argued that one should regard $r=2 m$, at a given $t$, as a single point: for this one has to violate the metric topology in the opposite sense, unacceptably in my view, by identifying points which appear to be finitely separated from one another. The remaining issue is to consider whether different values of $t$ at $r=2 m$ should be identified. The transformation 
between the Schwarzschild and Kruskal-Szekeres coordinates is

$$
u=(r / 2 m-1)^{1 / 2} e^{r / 4 m} \cosh t / 4 m, \quad v=(r / 2 m-1)^{1 / 2} e^{r / 4 m} \sinh t / 4 m .
$$

from which we easily see that for $r=2 m$ and all finite $t$ we get only the single sphere $u=v=0$. This is exactly what we would expect from the analogy with behaviour at axes. Incidentally, we may note that $r$ is a welldefined scalar function on the whole Kruskal-Szekeres manifold (though not a well-defined coordinate since its constant surfaces in general have two disconnected pieces). The future part of the horizon bounding the region we started with, $0<u=v<\infty$, is the topological product of a sphere and a line, and is the boundary $t \rightarrow \infty$ of the Schwarzschild coordinate patch, and the past part $0<u=-v<\infty$ is similarly $t \rightarrow-\infty$. Thus the points of different finite $t$ with $r=2 m$ have to be identified, while the limiting 'points' in the $(r, t)$ plane at $|t| \rightarrow \infty, r \rightarrow 2 m$, are lines. (The calculations of Antoci et al. in the usual $(t, r, \theta, \phi)$ coordinates, if the limit is at finite $t$, thus refer only to the sphere $u=v=0$ in the Kruskal picture: however, there will still be a divergent acceleration at other points of $r=2 m$ as argued above.)

Hence, it is hardly surprising that if one regards $r \geq 2 m$ as the coordinate patch, with the topology inherited from $H^{n}$, the coordinate transformation at $r=2 m$ to Kruskal or other coordinates in which the metric is regular on the horizon is a singular one and not 1-1. However, this is no more objectionable than, and indeed directly analogous to, transforming from polars to Cartesians at an axis in the Euclidean plane. Similar remarks apply to the other regular horizons considered. 